\title{
Strain relaxation in misfitting transition metal dichalcogenide monolayer superlattices: Wrinkling vs. misfit dislocation formation - Supporting Information
}

\author{
Yang Xia ${ }^{1}$, Ryan S. Davis ${ }^{1}$, and Mikko P. Haataja ${ }^{1,2}$ \\ ${ }^{1}$ Department of Mechanical and Aerospace Engineering, Princeton University, \\ Princeton, New Jersey 08544, United States \\ ${ }^{2}$ Princeton Institute for Science and Technology of Materials (PRISM), \\ Princeton University, Princeton, New Jersey 08544, United States
}

November 6, 2019

\section{Governing equations for binary TMD monolayer super- lattices}

In order to incorporate the chemical and elastic heterogeneities, an order parameter $\phi(\mathbf{r})$ is used to represent different chemical components within the superlattice $\left[\phi(\mathbf{r})=0\right.$, say, for $\mathrm{WS}_{2}$, and $\phi(\mathbf{r})=1$ for $\left.\mathrm{WSe}_{2}\right]$. In addition to $\phi(\mathbf{r})$, the presence of free boundaries is accounted for by introducing another order parameter $\psi(\mathbf{r})$, such that $\psi(\mathbf{r})=1(0)$ inside (outside) the monolayer.

As stated in the main text, the strain energy in our phase-field model is

$$
E_{\text {strain }}=\frac{1}{2} \int_{A} C_{i j k l}(\boldsymbol{r})\left[\epsilon_{i j}(\boldsymbol{r})-\epsilon_{i j}^{m i s}(\boldsymbol{r})\right]\left[\epsilon_{k l}(\boldsymbol{r})-\epsilon_{k l}^{m i s}(\boldsymbol{r})\right] d^{2} \mathbf{r}
$$

where $\mathbf{r}=[x, y]^{T}, C_{i j k l}(\mathbf{r})=\left\{[1-\phi(\mathbf{r})] C_{i j k l}^{(1)}+\phi(\mathbf{r}) C_{i j k l}^{(2)}\right\} \psi(\mathbf{r})$ denotes the local stiffness tensor, $\epsilon_{i j}(\mathbf{r})=\bar{\epsilon}_{i j}+\frac{1}{2}\left(\frac{\partial u_{i}(\mathbf{r})}{\partial r_{j}}+\frac{\partial u_{j}(\mathbf{r})}{\partial r_{i}}+\frac{\partial \omega(\mathbf{r})}{\partial r_{i}} \frac{\partial \omega(\mathbf{r})}{\partial r_{j}}\right)$ denotes the in-plane elastic strain tensor, and $\epsilon_{i j}^{m i s}(\mathbf{r})=$ $\left[\phi(\mathbf{r}) \epsilon_{i j}^{*}+\epsilon_{i j}^{d i s}(\boldsymbol{r})\right] \psi(\mathbf{r})$ denotes the local misfit strain tensor arising from both lattice mismatch and misfit dislocations. Furthermore, $\bar{\epsilon}_{i j}$ denotes the applied macroscopic strain, $\epsilon_{i j}^{*}$ denotes the misfit strain between TMDs 1 and 2 arising from lattice mismatch such that $\epsilon_{x x}^{*}=\frac{a_{2}-a_{1}}{a_{1}}, \epsilon_{y y}^{*}=\frac{b_{2}-b_{1}}{b_{1}}$, and $\epsilon_{x y}^{*}=0$, while $\epsilon_{i j}^{d i s}(\boldsymbol{r})$ is the dislocation-induced strain defined below in Eq. [2]. Additionally, $C_{i j k l}^{(1)}, C_{i j k l}^{(2)}, a_{1}, a_{2}, b_{1}$ and $b_{2}$ denote stiffness tensors and lattice constants of TMDs 1 and 2 corresponding to the $2 \mathrm{H}$ crystal structure, respectively. Finally, $u_{i}(\mathbf{r})$ and $\omega(\mathbf{r})$ denote the in-plane and out-of-plane displacements, respectively.

Dislocations in 3D phase field models can be described as misfitting platelets corresponding to the slipped regions within the crystal. ${ }^{1}$ Specifically, upon introducing a set of additional order parameters $\zeta_{q}(\boldsymbol{r})$, the effective misfit strain arising from the dislocations can be written

$$
\epsilon_{i j}^{d i s}(\boldsymbol{r})=\frac{\boldsymbol{b} \cdot \boldsymbol{n}}{2 d} \zeta(\boldsymbol{r})
$$


where $\boldsymbol{b}$ denotes the Burger's vector, $\boldsymbol{n}$ is the slip plane normal, and $d$ is the interplanar distance. In our quasi-2D model, the dislocation loops turn into linear segments with non-zero $\zeta(\boldsymbol{r})$, with the end points of the segment corresponding to two edge dislocations.

Now, the total energy of the bendable, substrate-supported sheet can be written

$$
\begin{aligned}
E_{\text {total }}= & E_{\text {strain }}+E_{\text {bend }}+E_{\text {sub }}=\frac{1}{2} \int_{A} C_{i j k l}(\boldsymbol{r})\left[\epsilon_{i j}(\boldsymbol{r})-\epsilon_{i j}^{m i s}(\boldsymbol{r})\right]\left[\epsilon_{k l}(\boldsymbol{r})-\epsilon_{k l}^{m i s}(\boldsymbol{r})\right] d^{2} \mathbf{r} \\
& +\int_{A} \frac{\kappa(\boldsymbol{r})}{2}\left\{\left(\nabla^{2} \omega(\boldsymbol{r})\right)^{2}+\left[(1-\nu(\boldsymbol{r})]\left[\left(\frac{\partial^{2} \omega(\boldsymbol{r})}{\partial x \partial y}\right)^{2}-\frac{\partial^{2} \omega(\boldsymbol{r})}{\partial x^{2}} \frac{\partial^{2} \omega(\boldsymbol{r})}{\partial y^{2}}\right]\right\} d^{2} \mathbf{r}\right. \\
& +k_{\text {eff }} \int_{A}\left[\omega(\boldsymbol{r})-\omega_{e q}\right]^{2} d^{2} \mathbf{r}
\end{aligned}
$$

where $\kappa(\mathbf{r})=\frac{Y(\mathbf{r}) h^{3}}{24\left(1-\nu(\mathbf{r})^{2}\right)}$ denotes the local bending rigidity, while $\nu(\mathbf{r})=\left\{[1-\phi(\mathbf{r})] \nu_{1}+\phi(\mathbf{r}) \nu_{2}\right\} \psi(\mathbf{r})$ denotes local Poisson's ratio. Furthermore, $Y(\mathbf{r})=\left\{[1-\phi(\mathbf{r})] Y_{1}+\phi(\mathbf{r}) Y_{2}\right\} \psi(\mathbf{r})$ denotes the local Young's modulus, and $h$ denotes the thickness of the sheet. Finally, $\nu_{1}, \nu_{2}, Y_{1}$ and $Y_{2}$ denote the Poisson ratios and Young's moduli of TMDs 1 and 2, respectively.

Given (1) the fixed spatial distribution of the chemical species within the monolayer superlattice [which control the local elastic moduli $C_{i j k l}(\mathbf{r})$ and $\kappa(\mathbf{r})$, and the misfit strain $\epsilon_{i j}^{m i s}(\mathbf{r})$ ], (2) spatial distribution of misfit dislocations, and (3) far-field boundary conditions (i.e., strain-controlled vs. stress-controlled boundaries), minimization of Eq. [3] yields the equilibrium configuration of the monolayer. More specifically, in the case of stress-free far-field boundary conditions adopted in the present work, Eq. [3] is minimized with respect to macroscopic strains, in-plane and outof-plane displacements $\left(\bar{\epsilon}_{i j}, u_{i}\right.$ and $\omega$, respectively). For strain-controlled boundary conditions, on the other hand, $\bar{\epsilon}_{i j}$ is prescribed.

\subsection{Dimensionless total energy}

To facilitate the numerical computations, the total energy of the system is rendered dimensionless by introducing $\tilde{\boldsymbol{r}}=\frac{\boldsymbol{r}}{W}, \tilde{\omega}=\frac{\omega}{W}, \tilde{\omega}_{e q}=\frac{\omega_{e q}}{W}, \tilde{k}_{e f f}=\frac{k_{e f f} W}{Y_{1}}, \tilde{C}_{i j k l}=\frac{C_{i j k l}}{Y_{1} W}, \tilde{\kappa}=\frac{\kappa}{Y_{1} W^{3}}$, and $\tilde{E}_{t o t a l}=\frac{E_{t o t a l}}{Y_{1} W^{3}}$ with $W$ denoting the superlattice period. The dimensionless total energy can now be expressed as

$$
\begin{aligned}
\tilde{E}_{t o t a l}= & \int_{A} \frac{1}{2} \tilde{C}_{i j k l}(\tilde{\boldsymbol{r}})\left[\epsilon_{i j}(\tilde{\boldsymbol{r}})-\epsilon_{i j}^{m i s}(\tilde{\boldsymbol{r}})\right]\left[\epsilon_{k l}(\tilde{\boldsymbol{r}})-\epsilon_{k l}^{m i s}(\tilde{\boldsymbol{r}})\right] d^{2} \tilde{\boldsymbol{r}} \\
& +\int_{A} \frac{\tilde{\kappa}(\tilde{\boldsymbol{r}})}{2}\left\{\left(\tilde{\nabla}^{2} \tilde{\omega}(\tilde{\boldsymbol{r}})\right)^{2}+[1-\nu(\tilde{\boldsymbol{r}})]\left[\left(\frac{\partial^{2} \tilde{\omega}(\tilde{\boldsymbol{r}})}{\partial \tilde{x} \partial \tilde{y}}\right)^{2}-\frac{\partial^{2} \tilde{\omega}(\tilde{\boldsymbol{r}})}{\partial \tilde{x}^{2}} \frac{\partial^{2} \tilde{\omega}(\tilde{\boldsymbol{r}})}{\partial \tilde{y}^{2}}\right]\right\} d^{2} \tilde{\boldsymbol{r}} \\
& +\tilde{k}_{e f f} \int_{A}\left[\tilde{\omega}(\tilde{\boldsymbol{r}})-\tilde{\omega}_{e q}\right]^{2} d^{2} \tilde{\boldsymbol{r}} .
\end{aligned}
$$

Therefore, in order to vary the superlattice period $W$ for a given binary TMD superlattice, it is more convenient to fix the dimensionless (numerical) superlattice period, and adjust the dimensionless parameters $\tilde{C}_{i j k l}, \tilde{\kappa}, \tilde{\omega}$ and $\tilde{k}_{e f f}$ accordingly.

\section{$1.2 \quad$ Eigenstrain method}

In order to efficiently relax the in-plane displacements and apply stress free boundary conditions in the simulations, an eigenstrain method pioneered by Wang et $a l^{2}{ }^{2}$ is employed. To this end, we 
first define $\Delta \tilde{C}_{i j k l}(\tilde{\boldsymbol{r}})=\tilde{C}_{i j k l}^{1}-\tilde{C}_{i j k l}(\tilde{\boldsymbol{r}})$ with $\tilde{C}_{i j k l}^{1}=\frac{C_{i j k l}^{1}}{Y_{1} W}$. A new effective eigenstrain tensor $\epsilon_{i j}^{0}(\tilde{\boldsymbol{r}})$ can be defined such that

$$
\tilde{C}_{i j k l}^{1} \epsilon_{k l}^{0}(\tilde{\boldsymbol{r}})=\tilde{C}_{i j k l}^{1} \epsilon_{k l}^{m i s}(\tilde{\boldsymbol{r}})+\Delta \tilde{C}_{i j k l}(\tilde{\boldsymbol{r}})\left[\epsilon_{k l}(\tilde{\boldsymbol{r}})-\epsilon_{k l}^{m i s}(\tilde{\boldsymbol{r}})\right]
$$

With the above definition of $\epsilon_{i j}^{0}(\tilde{\boldsymbol{r}})$, one can relate the strain energy of the inhomogeneous system to a homogeneous one: ${ }^{2}$

$$
\begin{aligned}
\tilde{E}_{\text {strain }}= & \frac{1}{2} \int_{A} \tilde{C}_{i j k l}^{1}\left[\epsilon_{i j}(\tilde{\boldsymbol{r}})-\epsilon_{i j}^{0}(\tilde{\boldsymbol{r}})\right]\left[\epsilon_{k l}(\tilde{\boldsymbol{r}})-\epsilon_{k l}^{0}(\tilde{\boldsymbol{r}})\right] d^{2} \tilde{\boldsymbol{r}} \\
& +\frac{1}{2} \int_{A}\left[\tilde{C}_{i j m n}^{1} \Delta \tilde{S}_{m n p q}(\tilde{\boldsymbol{r}}) \tilde{C}_{p q k l}^{1}\right]\left[\epsilon_{i j}^{0}(\tilde{\boldsymbol{r}})-\epsilon_{i j}^{m i s}(\tilde{\boldsymbol{r}})\right]\left[\epsilon_{k l}^{0}(\tilde{\boldsymbol{r}})-\epsilon_{k l}^{m i s}(\tilde{\boldsymbol{r}})\right] d^{2} \tilde{\boldsymbol{r}} \\
\tilde{E}_{\text {bend }}= & \int_{A} \frac{\tilde{\kappa}(\tilde{\boldsymbol{r}})}{2}\left\{\left(\tilde{\nabla}^{2} \tilde{\omega}(\tilde{\boldsymbol{r}})\right)^{2}+[1-\nu(\tilde{\boldsymbol{r}})]\left[\left(\frac{\partial^{2} \tilde{\omega}(\tilde{\boldsymbol{r}})}{\partial \tilde{x} \partial \tilde{y}}\right)^{2}-\frac{\partial^{2} \tilde{\omega}(\tilde{\boldsymbol{r}})}{\partial \tilde{x}^{2}} \frac{\partial^{2} \tilde{\omega}(\tilde{\boldsymbol{r}})}{\partial \tilde{y}^{2}}\right]\right\} d^{2} \tilde{\boldsymbol{r}},
\end{aligned}
$$

where $\tilde{E}_{\text {strain }}=\frac{E_{\text {strain }}}{Y_{1} W^{3}}, \tilde{E}_{b e n d}=\frac{E_{\text {bend }}}{Y_{1} W^{3}}$, and $\Delta \tilde{S}_{i j k l}(\tilde{\boldsymbol{r}})$ denotes the inverse tensor of $\Delta \tilde{C}_{i j k l}(\tilde{\boldsymbol{r}})$.

Now, the first integral in Eq. [6] can be regarded as the strain energy in an elastically homogeneous system with the as-of-yet unknown eigenstrain $\epsilon_{i j}^{0}(\tilde{\boldsymbol{r}})$ acting as an effective misfit strain. In addition, the second integral in Eq. [6] has no dependence on the in-plane strain tensor $\epsilon_{i j}(\tilde{\boldsymbol{r}})$. Therefore, once $\epsilon_{i j}^{0}(\tilde{\boldsymbol{r}})$ has been determined, the in-plane strains can be readily obtained following the approach of Khachaturyan. ${ }^{3}$ Explicitly, the equations for the macroscopic strain $\bar{\epsilon}_{i j}$, eigenstrain $\epsilon_{i j}^{0}(\tilde{\boldsymbol{r}})$, in-plane displacements $\tilde{u}_{i}(\tilde{\boldsymbol{r}})$, and out-of-plane displacement $\tilde{\omega}(\tilde{\boldsymbol{r}})$ for a system in mechanical equilibrium are given by

$$
\begin{aligned}
& \bar{\epsilon}_{i j}=\frac{1}{A} \int_{A}\left[\epsilon_{i j}^{o}(\tilde{\boldsymbol{r}})-\frac{1}{2} \frac{\partial \omega}{\partial r_{i}} \frac{\partial \omega}{\partial r_{j}}\right] d^{2} \tilde{\boldsymbol{r}} \\
& \frac{\delta \tilde{E}_{t o t a l}}{\delta \epsilon_{i j}^{0}(\tilde{\boldsymbol{r}})}=\frac{\delta \tilde{E}_{s t r a i n}}{\delta \epsilon_{i j}^{0}(\tilde{\boldsymbol{r}})}=\tilde{C}_{i j k l}^{(1)} \Delta \tilde{S}_{k l m n}(\tilde{\boldsymbol{r}}) \tilde{C}_{m n s t}^{(1)}\left[\epsilon_{s t}^{0}(\tilde{\boldsymbol{r}})-\epsilon_{s t}^{m i s}(\tilde{\boldsymbol{r}})\right]-\tilde{C}_{i j k l}^{(1)}\left[\epsilon_{k l}(\tilde{\boldsymbol{r}})-\epsilon_{k l}^{m i s}(\tilde{\boldsymbol{r}})\right]=0 ; \\
& \widehat{u}_{i}(\boldsymbol{k})=-i G_{i j}(\boldsymbol{k}) \tilde{C}_{j k l m}^{1} \widehat{\epsilon}_{l m}^{0}(\boldsymbol{k}) k_{k}+G_{i j}(\boldsymbol{k}) \tilde{C}_{j k l m}^{1} \widehat{N}_{k l m}(\boldsymbol{k}) ; \\
& \frac{\delta \tilde{E}_{t o t a l}}{\delta \tilde{\omega}(\tilde{\boldsymbol{r}})}=-2 \frac{\partial}{\partial \tilde{r}_{i}}\left\{\left[\tilde{C}_{i j k l}^{1}-\Delta \tilde{C}_{i j k l}(\tilde{\boldsymbol{r}})\right] \frac{\partial \tilde{\omega}}{\partial \tilde{r}_{j}}\left[\epsilon_{k l}(\tilde{\boldsymbol{r}})-\epsilon_{k l}^{m i s}(\tilde{\boldsymbol{r}})\right]\right\} \\
& +\frac{\partial^{2}}{\partial \tilde{x}^{2}}\left[\kappa(\tilde{\boldsymbol{r}}) \frac{\partial^{2} \tilde{\omega}(\tilde{\boldsymbol{r}})}{\partial \tilde{x}^{2}}+\tilde{\kappa}(\tilde{\boldsymbol{r}}) \frac{\partial^{2} \tilde{\omega}(\tilde{\boldsymbol{r}})}{\partial \tilde{y}^{2}}\right]+\frac{\partial^{2}}{\partial \tilde{y}^{2}}\left[\tilde{\kappa}(\tilde{\boldsymbol{r}}) \frac{\partial^{2} \tilde{\omega}(\tilde{\boldsymbol{r}})}{\partial \tilde{x}^{2}}+\tilde{\kappa}(\tilde{\boldsymbol{r}}) \frac{\partial^{2} \tilde{\omega}(\tilde{\boldsymbol{r}})}{\partial \tilde{y}^{2}}\right] \\
& +2 \frac{\partial^{2}}{\partial \tilde{x} \partial \tilde{y}}\left[\tilde{\kappa}(\tilde{\boldsymbol{r}})(1-\nu(\tilde{\boldsymbol{r}})) \frac{\partial^{2} \tilde{\omega}(\tilde{\boldsymbol{r}})}{\partial \tilde{x} \partial \tilde{y}}\right]-\frac{\partial^{2}}{\partial \tilde{x}^{2}}\left[\tilde{\kappa}(\tilde{\boldsymbol{r}})(1-\nu(\tilde{\boldsymbol{r}})) \frac{\partial^{2} \tilde{\omega}(\tilde{\boldsymbol{r}})}{\partial \tilde{y}^{2}}\right] \\
& -\frac{\partial^{2}}{\partial \tilde{y}^{2}}\left[\tilde{\kappa}(\tilde{\boldsymbol{r}})(1-\nu(\tilde{\boldsymbol{r}})) \frac{\partial^{2} \tilde{\omega}(\tilde{\boldsymbol{r}})}{\partial \tilde{x}^{2}}\right]+2 \tilde{k}_{e f f}\left[\omega(\tilde{\boldsymbol{r}})-\tilde{\omega}_{e q}(\tilde{\boldsymbol{r}})\right]=0
\end{aligned}
$$

respectively, where $\widehat{\epsilon}_{i j}^{0}(\boldsymbol{k})$ denotes the Fourier transform of $\epsilon_{i j}^{0}(\tilde{\boldsymbol{r}}), \Omega_{i j}(\boldsymbol{n})^{-1}=\tilde{C}_{i j k l}^{1} n_{k} n_{l}, \boldsymbol{n}=\boldsymbol{k} /|k|$, $\widehat{u}_{i}(\boldsymbol{k})$ denotes the Fourier transform of $\tilde{u}_{i}(\tilde{\boldsymbol{r}})=\frac{u_{i}(\boldsymbol{r})}{W}, G_{i j}(\boldsymbol{k})=\Omega_{i j}(\boldsymbol{n}) / k^{2}$, and

$$
\widehat{N}_{k l m}(\boldsymbol{k})=\left\{\frac{\partial \tilde{\omega}}{\partial \tilde{r}_{k}} \frac{\partial^{2} \tilde{\omega}}{\partial \tilde{r}_{l} \partial \tilde{r}_{m}}\right\}_{\boldsymbol{k}},
$$

with \{\}$_{\boldsymbol{k}}$ denoting the Fourier transform. 


\section{Numerical relaxation scheme}

We first note that Eq. [10] provides a closed-form, analytical solution for $\tilde{u}_{i}(\tilde{\boldsymbol{r}})$ by way of a simple Fourier transform. However, Eqs. [9] and [10] together in fact comprise an integro-differential equation for $\epsilon_{i j}^{0}(\tilde{\boldsymbol{r}})$, while Eq. [11] is a non-linear partial differential equation with non-constant coefficients. In order to numerically solve them, the following iterative scheme was implemented:

1. Under the assumption of negligible diffusion of the chemical species, the order parameters $\phi(\tilde{\boldsymbol{r}})$ and $\psi(\tilde{\boldsymbol{r}})$ are fixed and do not vary during the numerical relaxation process.

2. Locations of the misfit dislocations (as encoded by $\zeta(\boldsymbol{r})$ ) and their Burger's vectors are fixed at the beginning of the simulation, and they remain stationary during the simulation.

3. Compute $\bar{\epsilon}_{i j}$ from Eq. [8].

4. Recast Eq. [9] as the steady-state solution of a fictitious time-dependent system, and equilibrate $\epsilon_{i j}^{0}(\tilde{\boldsymbol{r}})$ by solving

$$
\frac{\partial^{2} \epsilon_{i j}^{0}(\tilde{\boldsymbol{r}}, \tilde{t})}{\partial \tilde{t}^{2}}+\left(\gamma_{0 ; \epsilon}-\gamma_{1 ; \epsilon} \nabla^{2}\right) \frac{\partial \epsilon_{i j}^{0}(\tilde{\boldsymbol{r}}, \tilde{t})}{\partial \tilde{t}}=-\alpha_{\epsilon}^{2} \frac{\delta \tilde{E}_{t o t a l}}{\delta \epsilon_{i j}^{0}(\tilde{\boldsymbol{r}}, \tilde{t})},
$$

where $\gamma_{0 ; \epsilon}$ denotes a uniform damping parameter, $\gamma_{1 ; \epsilon}$ is a damping parameter that preferentially suppresses large wavenumber oscillations, $\alpha_{\epsilon}$ is the effective wave speed, and $\tilde{t}$ represents a fictitious dimensionless "time" in the relaxation scheme.

5. Compute $\tilde{u}_{i}(\tilde{\boldsymbol{r}})$ from Eq. [10].

6. Evaluate $\epsilon_{i j}(\tilde{\boldsymbol{r}})=\bar{\epsilon}_{i j}+\frac{1}{2}\left[\frac{\partial \tilde{u}_{i}(\tilde{\boldsymbol{r}})}{\partial \tilde{r}_{j}}+\frac{\partial \tilde{u}_{j}(\tilde{\boldsymbol{r}})}{\partial \tilde{r}_{i}}+\frac{\partial \tilde{\omega}(\tilde{\boldsymbol{r}})}{\partial \tilde{r}_{i}} \frac{\partial \tilde{\omega}(\tilde{\boldsymbol{r}})}{\partial \tilde{r}_{j}}\right]$.

7. Iterate $\tilde{\omega}(\tilde{\boldsymbol{r}})$ to equilibrium using

$$
\frac{\partial^{2} \tilde{\omega}(\tilde{\boldsymbol{r}}, \tilde{t})}{\partial \tilde{t}^{2}}+\left(\gamma_{0 ; \tilde{\omega}}-\gamma_{1 ; \tilde{\omega}} \nabla^{2}\right) \frac{\partial \tilde{\omega}(\tilde{\boldsymbol{r}}, \tilde{t})}{\partial \tilde{t}}=-\alpha_{\tilde{\omega}}^{2} \frac{\delta \tilde{E}_{t o t a l}}{\delta \tilde{\omega}(\tilde{\boldsymbol{r}}, \tilde{t})}
$$

where $\gamma_{0 ; \tilde{\omega}}, \gamma_{1, \tilde{\omega}}$, and $\alpha_{\tilde{\omega}}$ again denote effective damping parameters and wave speed. During the numerical simulations, Eq. [14] is first iterated until $\tilde{\omega}(\tilde{\boldsymbol{r}})$ converges, then return to Step 3 and repeat until both $\epsilon_{i j}^{0}(\tilde{\boldsymbol{r}})$ and $\tilde{\omega}(\tilde{\boldsymbol{r}})$ converge.

\section{Parameter values for TMDs}

The elastic moduli and lattice constants employed in this work are summarized in Table S1. The dynamic parameters are set to $\alpha_{\epsilon}=0.5, \gamma_{0 ; \epsilon}=1, \gamma_{1 ; \epsilon}=1, \alpha_{\tilde{\omega}}=5, \gamma_{0 ; \tilde{\omega}}=1$, and $\gamma_{1 ; \tilde{\omega}}=1$ with a discrete grid spacing $\Delta \tilde{x}=1$ and time step $\Delta \tilde{t}=0.025$. The order parameter $\phi(\tilde{\boldsymbol{r}})$ is set to be 0.05 for TMD 1 and 1 for TMD 2, while the interface is described via a simple hyperbolic tangent function to yield a diffuse interface. [We cannot set $\phi(\tilde{\boldsymbol{r}})=0$ anywhere in the system, otherwise $\Delta \tilde{C}_{i j k l}(\tilde{\boldsymbol{r}})=0$ and $\Delta \tilde{S}_{i j k l}(\tilde{\boldsymbol{r}})$ becomes singular. Therefore, the lower limit of $\phi(\tilde{\boldsymbol{r}})$ is set to be 0.05 in our numerical work.] Hence, due to the presence of a lower limit of $\phi(\tilde{\boldsymbol{r}})$ and the diffuse interface, a $10 \%$ uncertainty is assigned to our numerical computations. As the specific value of $\tilde{\omega}_{e q}$ is arbitrary, in our numerical calculations we have set $\tilde{\omega}_{e q}=15$, except for the simulations reported in Fig. 5 in the main text, for which $\tilde{\omega}_{e q}=25$. 
Table S1: Young's modulus $Y$, Poisson ratio $\nu,{ }^{5}$ and lattice constants $a$ and $b^{6}$ of select TMDs.

\begin{tabular}{ccccc}
\hline \hline TMDs & $Y\left(\mathrm{eV} / \mathrm{nm}^{3}\right)$ & $\nu$ & $a(n m)$ & $b(\mathrm{~nm})$ \\
\hline $\mathrm{MoS}_{2}$ & 1196 & 0.25 & 0.3183 & 0.5513 \\
$\mathrm{MoSe}_{2}$ & 998 & 0.23 & 0.3318 & 0.5747 \\
$\mathrm{MoTe}_{2}$ & 762 & 0.24 & 0.3550 & 0.6149 \\
$\mathrm{WS}_{2}$ & 1340 & 0.22 & 0.3182 & 0.5511 \\
$\mathrm{WSe}_{2}$ & 1114 & 0.19 & 0.3315 & 0.5744 \\
$\mathrm{WTe}_{2}$ & 830 & 0.18 & 0.3552 & 0.6154 \\
\hline \hline
\end{tabular}

Table S 2: Wrinkling amplitude $H$ and wavelength $\lambda$ obtained from numerical computations corresponding to different superlattice periods $W$ for the substrate-supported $\mathrm{WS}_{2} / \mathrm{WSe}_{2}$ system.

\begin{tabular}{||ccc||}
\hline$W(n m)$ & $H(n m)$ & $\lambda(n m)$ \\
\hline \hline 29.07 & 1.46 & 23.25 \\
\hline 20.55 & 1.55 & 24.6 \\
\hline 14.53 & 1.47 & 23.25 \\
\hline 11.87 & 1.47 & 23.75 \\
\hline 10.28 & 1.47 & 24.65 \\
\hline 9.19 & 1.40 & 23.9 \\
\hline 8.39 & 1.35 & 23.5 \\
\hline
\end{tabular}

Finally, upon employing a substrate interaction coefficient $k_{e f f}=0.069 \mathrm{eV} / \mathrm{nm}^{4}$, the numerical computations yield $H \approx 1 \mathrm{~nm}$ and $\lambda \approx 24 \mathrm{~nm}$ (Table S2), in excellent quantitative agreement with experimental observations. ${ }^{7}$

\section{Semi-analytical model for out-of-plane deflections}

In order to better capture the dependence of $E_{b e n d}$ at large superlattice periods $W$, as discussed in the main text, a simple empirical fitting form $\tilde{\omega}(\tilde{\boldsymbol{r}})-\tilde{\omega}_{e q}=\sin \left(\frac{\pi \tilde{y}}{80}\right)\left[A \exp \left(\frac{-|\tilde{x}-100|^{2.5}}{B}\right)+C\right]$ is employed. Numerically obtained $\tilde{\omega}(\tilde{\boldsymbol{r}})$ and fits at several different $W$ are shown in Figs. S1 and S2. In addition, the three fitting parameters are shown in Fig. S3 as functions of $W$. We note that, given the dependence of $A, B$ and $C$ on $W$, it follows that $E_{b e n d} / L \sim 1 / W$ asymptotically for large $W$, as can be verified with the aid of Eq. [4].

\section{$5 \quad$ Extra strain and bending energy around interfaces}

As discussed in the main text (and shown in Figs. S4 and S5), the elastic strain $\epsilon_{y y}(\tilde{\boldsymbol{r}})-\epsilon_{y y}^{m i s}(\tilde{\boldsymbol{r}})$ in the wrinkled state is smaller than $1 \%$ inside the stripes, which is what we expect from the scaling theory. However, it is impossible to have a constant wrinkling amplitude within the compressive areas and a vanishingly small one within the tensile regions due to the continuity of $\tilde{\omega}(\tilde{\boldsymbol{r}})$ across 


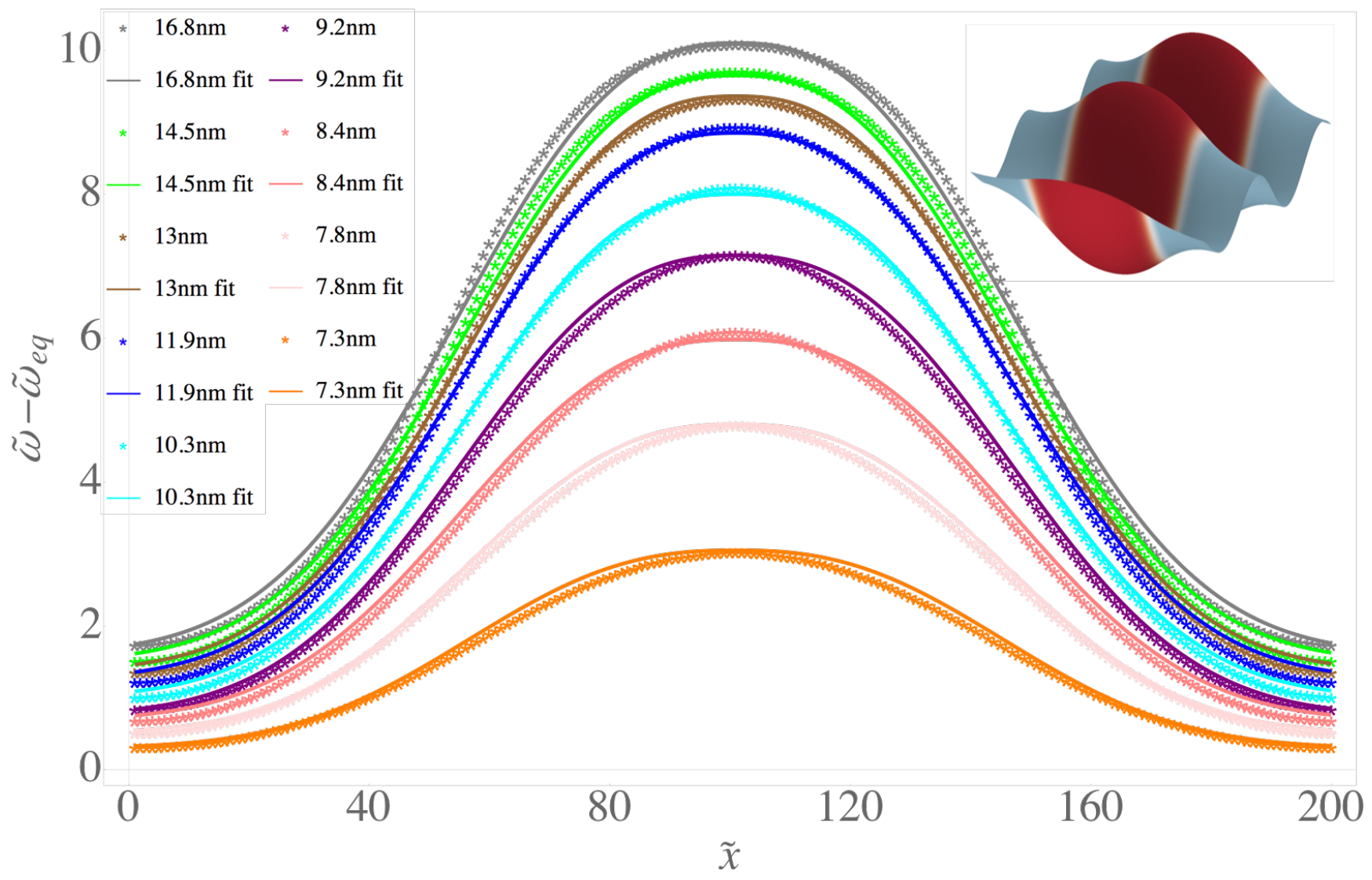

Figure S1: Fitting of $\tilde{\omega}(\tilde{\boldsymbol{r}})$ in the $x$ direction vs. numerically obtained profiles corresponding to different widths $W$ in symmetric superlattices $(\alpha=1 / 2)$. The inset displays a representative 3D configuration of the system. 


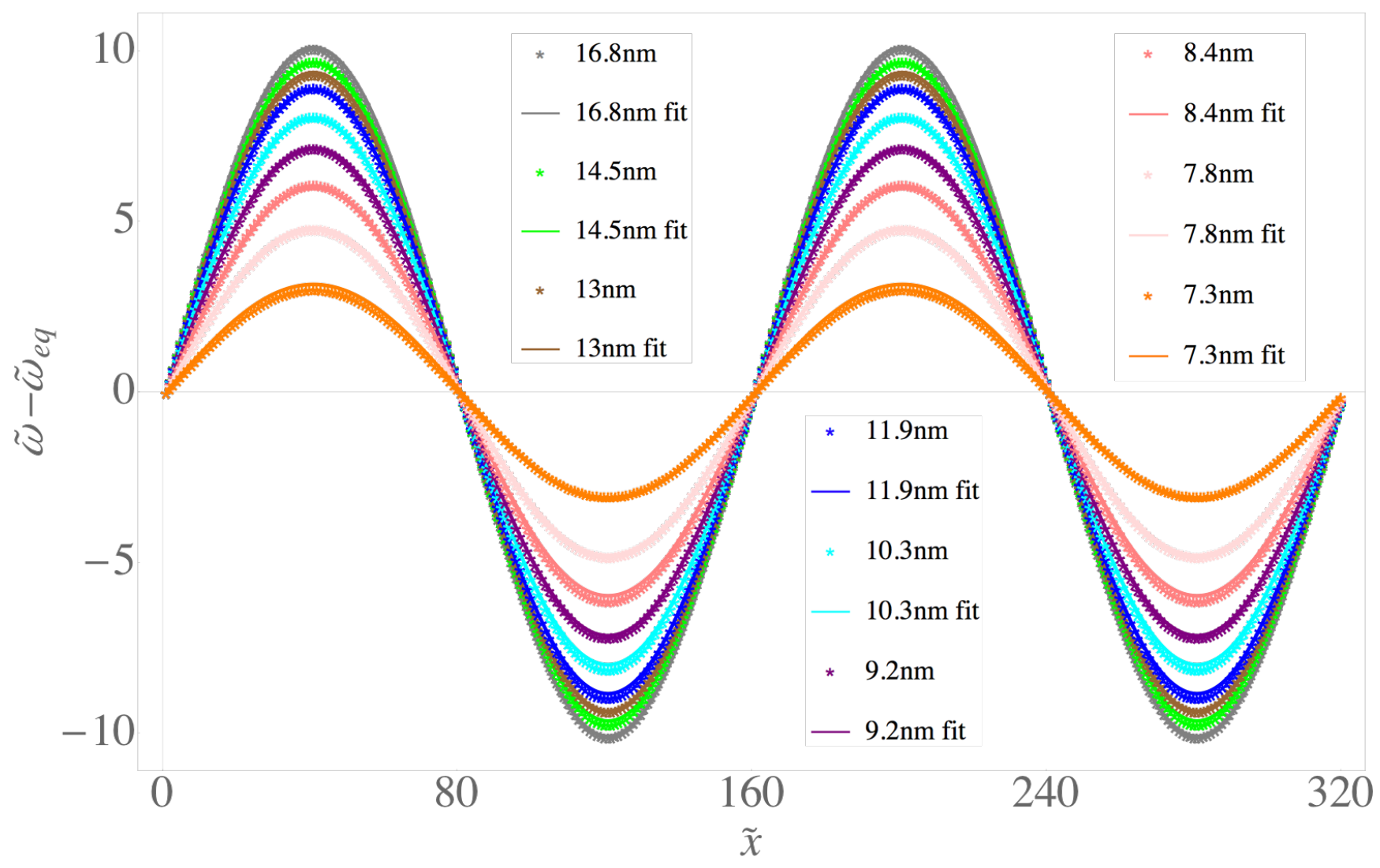

Figure S 2: Fitting of $\tilde{\omega}(\tilde{\boldsymbol{r}})$ in the $y$ direction vs. numerically obtained profiles corresponding to different widths $W$ in symmetric superlattices $(\alpha=1 / 2)$.
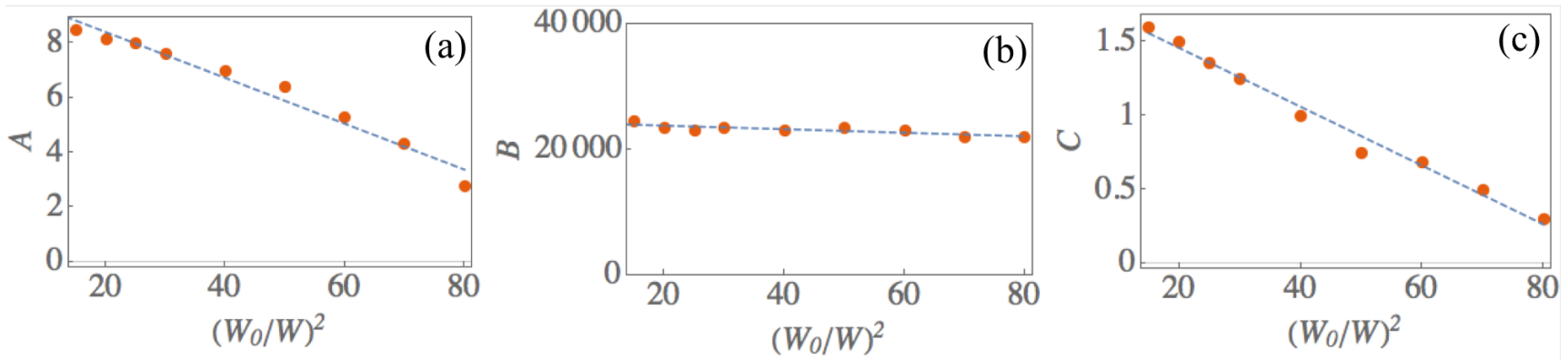

Figure S3: Fitting parameters (a) $A$, (b) $B$ and (c) $C$ versus $\left(W_{0} / W\right)^{2}$. 
(a)

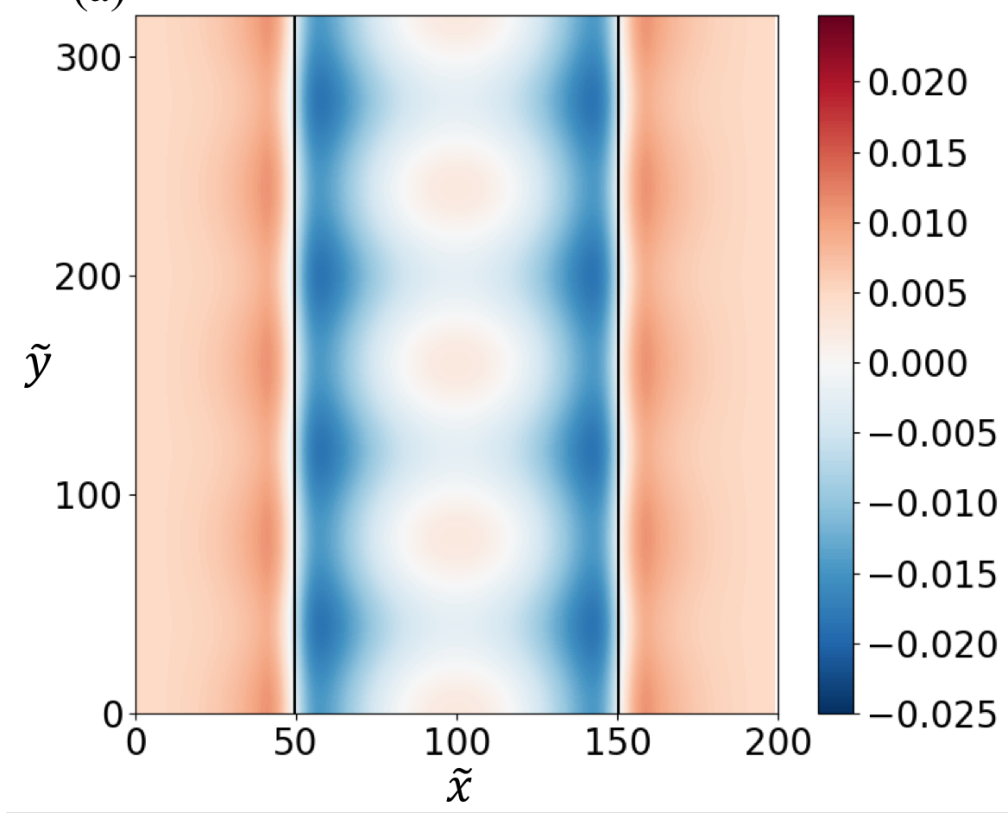

(b)

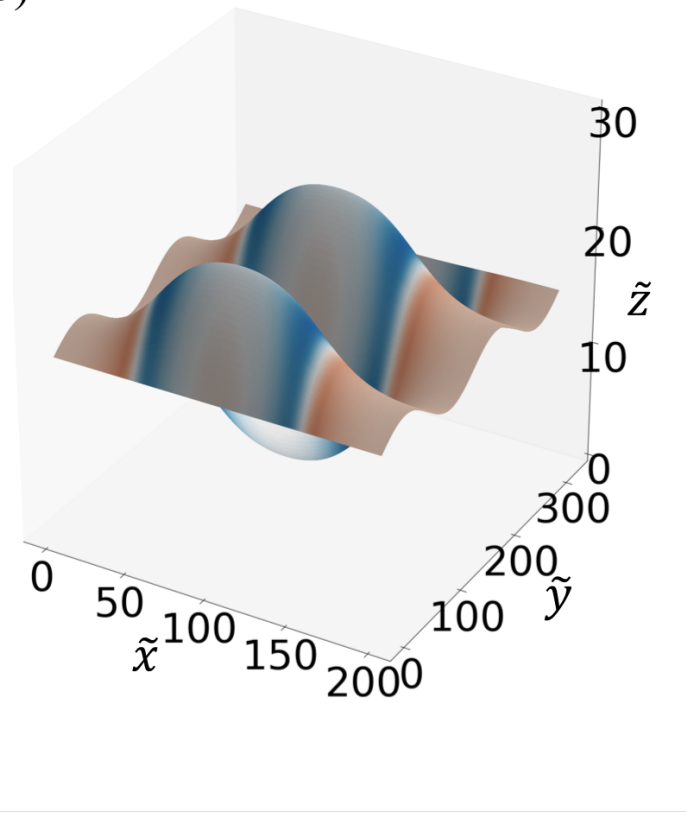

Figure S4: Elastic strain $\epsilon_{y y}(\tilde{\boldsymbol{r}})-\epsilon_{y y}^{m i s}(\tilde{\boldsymbol{r}})$ in a wrinkled, free-standing superlattice. (a) Top view. (b) $3 \mathrm{D}$ view. $W=14.5 \mathrm{~nm}$.

the interfaces. Hence, there is some remnant elastic strain around the interfaces, which results in additional strain and bending energy in those regions as compared with our scaling theory.

Now, as per our empirical fitting form $\tilde{\omega}(\tilde{\boldsymbol{r}})$, the ratio between the wrinkle amplitudes in compressive and tensile regions becomes a constant $R \approx 3$ when $W \rightarrow \infty$. Therefore, we can modify our scaling theory by accounting for the presence of bending deformations within the tensile regions to yield

$$
\begin{aligned}
\epsilon_{\text {com }} & =-\frac{2 \alpha \beta}{1+2 \alpha \beta}\left(\epsilon_{m i s}-\frac{2 H^{2} \pi^{2}}{\lambda^{2}}+\frac{2 H^{2} \pi^{2}}{R^{2} \lambda^{2}}\right) ; \\
\epsilon_{\text {ten }} & =\frac{1}{1+2 \alpha \beta}\left(\epsilon_{m i s}-\frac{2 H^{2} \pi^{2}}{\lambda^{2}}+\frac{2 H^{2} \pi^{2}}{R^{2} \lambda^{2}}\right),
\end{aligned}
$$

and the total deformation energy to be

$E_{\text {tot; wrinkle }}=W L\left[\frac{Y h \alpha \beta}{1+2 \alpha \beta}\left(\epsilon_{m i s}-\frac{2 H^{2} \pi^{2}}{\lambda^{2}}+\frac{2 H^{2} \pi^{2}}{R^{2} \lambda^{2}}\right)^{2}+\frac{H^{2} h^{3} \pi^{4} Y\left(1+\frac{\alpha \beta}{R^{2}}\right)}{3 \lambda^{4}\left(1-\nu^{2}\right)}+\frac{H^{2} k_{e f f}\left(1+\frac{\alpha}{R^{2}}\right)}{2}\right]$

In the case of free-standing monolayers, $k_{\text {eff }}=0$, and following the steps in the main text, we obtain

$$
\frac{E_{t o t ; w r i n k l e}}{W L}=\frac{Y h^{3} \pi^{2} \epsilon_{m i s}\left(\alpha \beta+R^{2}\right)}{6 \lambda^{2}\left(1-\nu^{2}\right)\left(R^{2}-1\right)}+O\left(\frac{h^{5}}{\lambda^{4}}\right)
$$

while in the case of substrate-supported superlattices,

$$
\begin{aligned}
\frac{E_{\text {tot; wrinkle }}}{W L} & =\frac{\epsilon_{\text {mis }}}{R^{2}-1} \sqrt{\frac{k_{e f f} Y h^{3}\left(\alpha+R^{2}\right)\left(\alpha \beta+R^{2}\right)}{6\left(1-\nu^{2}\right)}} \\
& -\frac{\left[2 \alpha^{3} \beta^{2}+R^{4}+\alpha R^{2}\left(1+\beta+2 \beta R^{2}\right)+\alpha^{2} \beta\left(1+2 R^{2}+2 \beta R^{2}\right)\right] h^{2} k_{e f f}}{24 \alpha \beta\left(1-\nu^{2}\right)\left(R^{2}-1\right)^{2}} .
\end{aligned}
$$


(a)

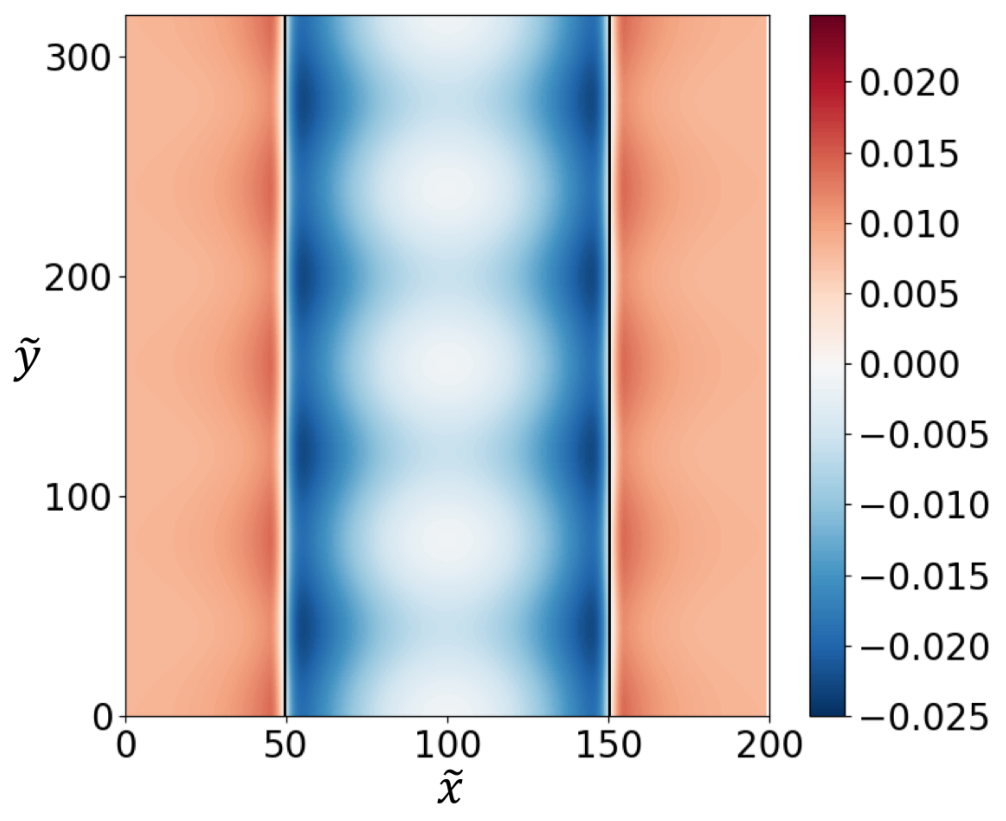

(b)

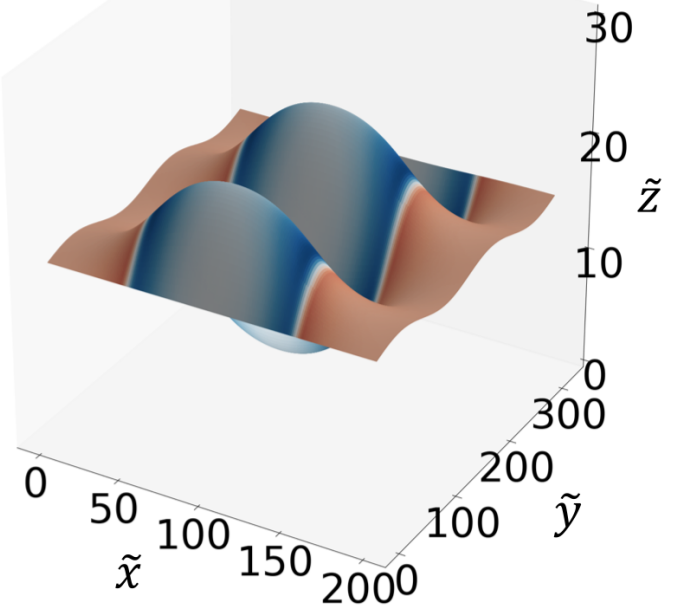

Figure S5: Elastic strain $\epsilon_{y y}(\tilde{\boldsymbol{r}})-\epsilon_{y y}^{m i s}(\tilde{\boldsymbol{r}})$ in a wrinkled, substrate-supported superlattice. (a) Top view. (b) $3 \mathrm{D}$ view. $W=14.5 \mathrm{~nm}$.

Hence, when $R^{2}>>1$, the results from the scaling theory (which assumes $R \rightarrow \infty$ ) still hold.

\section{Different binary superlattice width ratios}

All numerical computations and semi-analytical model results presented in the main text focus on the symmetric, equal-width TMD superlattice case $(\alpha=1 / 2)$. As superlattices with varying $\alpha$ can be readily synthesized, ${ }^{7}$ we have also employed $\alpha=1$ in the numerical computations to verify the robustness of our main conclusions.

To this end, we find that in the case of the free-standing superlattices, our data can now be fitted to $\tilde{\omega}(\tilde{\boldsymbol{r}})-\tilde{\omega}_{e q}=\sin \left(\frac{\pi \tilde{y}}{80}\right)\left[A_{1} \exp \left(\frac{-|\tilde{x}-150|^{2.4}}{B_{1}}\right)+C_{1}\right]$ (cf. Fig. S6). Furthermore, the fitting parameters $A^{\prime}=10.9-0.0499\left(\frac{W_{0}}{W}\right)^{2}, B^{\prime}=20600+14.7\left(\frac{W_{0}}{W}\right)^{2}$, and $C^{\prime}=0.523-0.0064\left(\frac{W_{0}}{W}\right)^{2}$ are linearly dependent on $\left(W_{0} / W\right)^{2}$, as shown in Fig. S7. With these empirical expressions for $\tilde{\omega}(\tilde{\boldsymbol{r}})$, $E_{\text {bend }}$ can again be readily evaluated numerically. As shown in Fig. S8(a), $E_{\text {bend }} / L$ calculated from the semi-analytical approach matches well with data from full numerical relaxation of both in-plane and out-of-plane deformations. Furthermore, given the dependence of $A^{\prime}, B^{\prime}$ and $C^{\prime}$ on $W$, it follows that $E_{\text {bend }} / L \sim 1 / W$ asymptotically for large $W$. Together with the observation that $E_{\text {strain }} / L \sim 1 / W$ [cf. Figs. S8(a) and S8(b)], this result implies that $E_{\text {total }} / L \sim 1 / W$ for $\alpha=1$, in quantitative agreement with the symmetric binary superlattice $(\alpha=1 / 2)$.

In the case of substrate-supported superlattices, on the other hand, $E_{\text {total }}, E_{\text {strain }}$ and $\Delta E$ obtained from full numerical relaxation of both in-plane and out-of-plane deformations with $\alpha=1$ are shown in Fig. S8(c). Again, it can be seen that $E_{\text {total }} \sim W$, in quantitative agreement with the symmetric binary superlattice $(\alpha=1 / 2)$. 


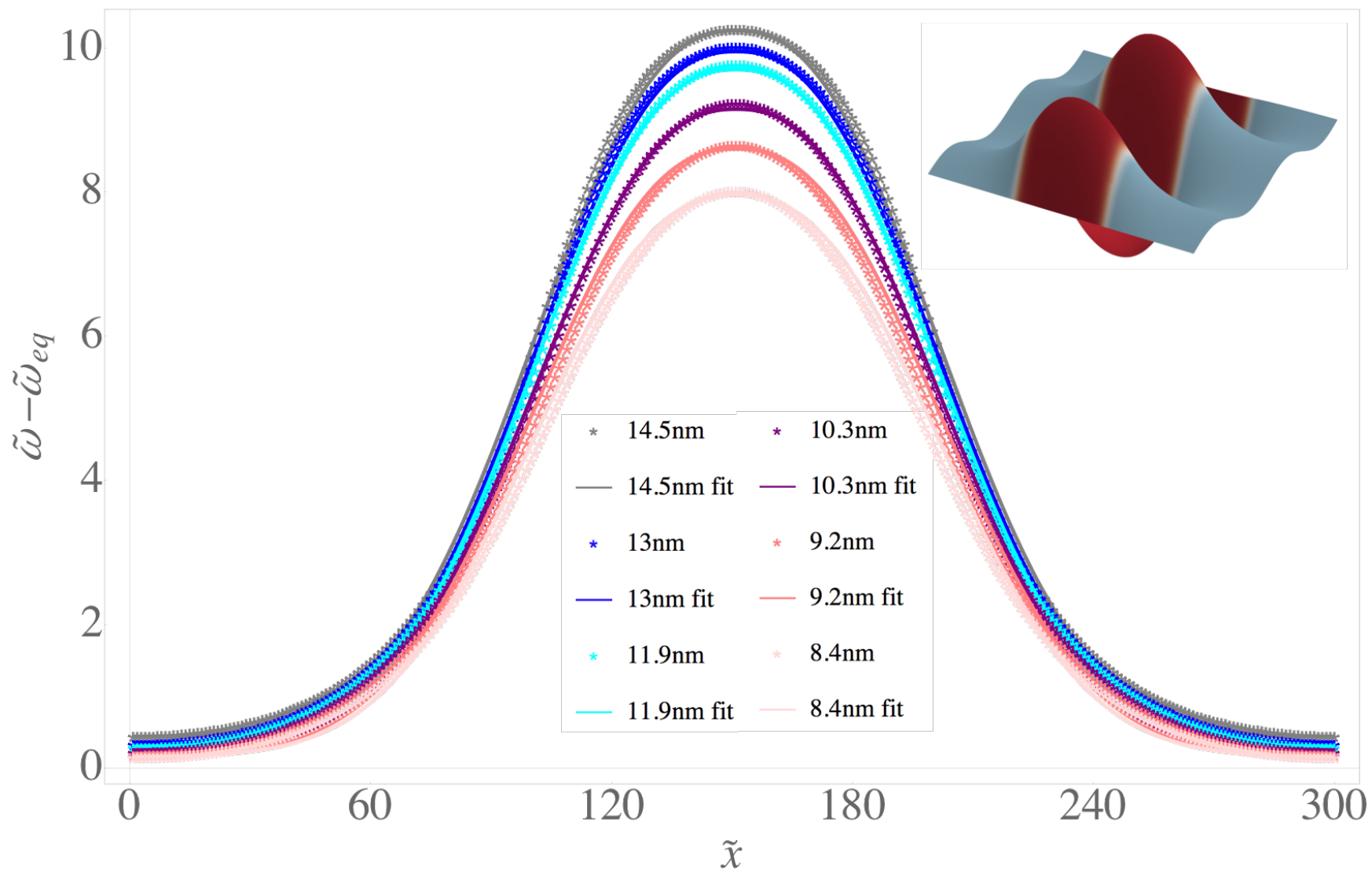

Figure S6: Fitting of $\tilde{\omega}(\tilde{\boldsymbol{r}})$ in the $x$ direction vs. numerically obtained profiles corresponding to different widths $W$ in asymmetric superlattices $(\alpha=1)$. The inset displays a representative 3D configuration of the system.
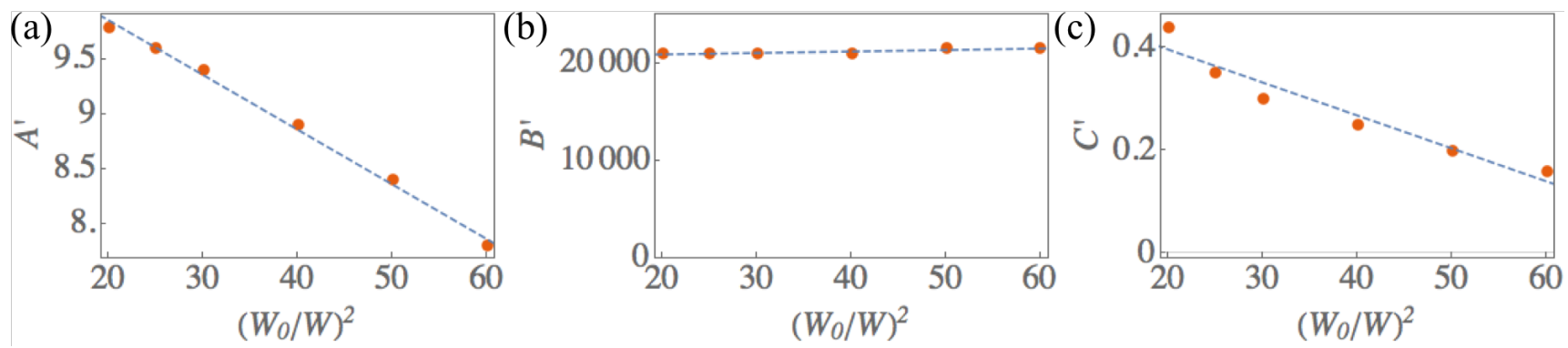

Figure S7: Fitting parameters (a) $A^{\prime}$, (b) $B^{\prime}$ and (c) $C^{\prime}$ versus $\left(W_{0} / W\right)^{2}$ for $\alpha=1$. 

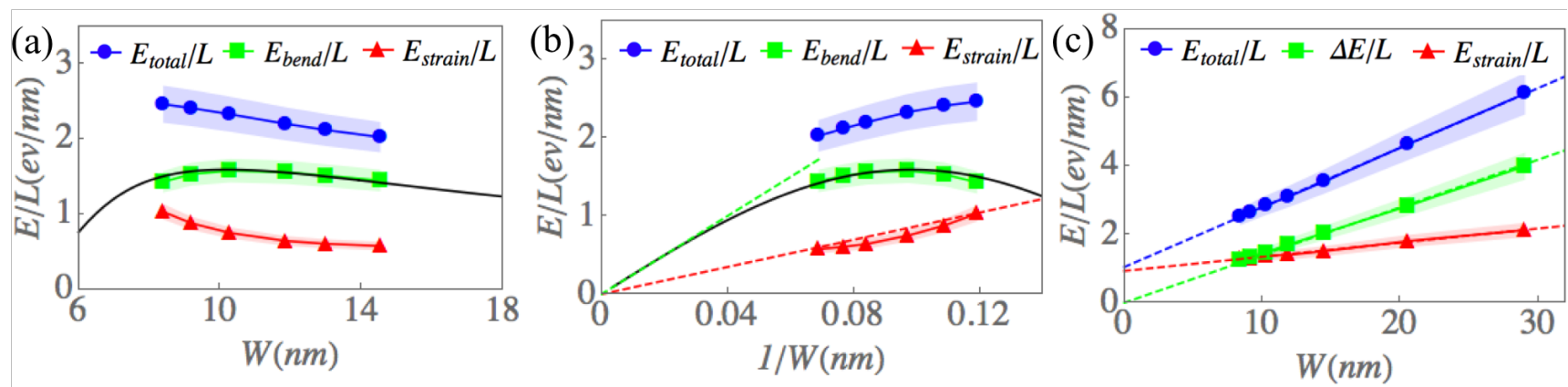

Figure S8: Energy of asymmetric $(\alpha=1)$, free-standing $\mathrm{WS}_{2} / \mathrm{WSe}_{2}$ superlattices from simulations versus (a) $W$ and (b) $1 / W$. (c) Energy of asymmetric, substrate-supported $\mathrm{WS}_{2} / \mathrm{WSe}_{2}$ superlattice from simulations versus $W . \Delta E=E_{\text {total }}-E_{\text {sub }}$. The black solid line is $E_{\text {bend }} / L$ calculated from the semi-analytical model. The dashed lines are fits while the gray areas represent estimated $10 \%$ confidence intervals.

\section{Scaling analysis of ternary superlattices}

Finally, we extend the scaling analysis of binary superlattices in the main text to ternary ones. To this end, we assume that stripe 1 is under tension and remains planar, while stripes 2 and 3 are under misfit-induced compression. Due to the compressive stresses, regions 2 and 3 wrinkle with wavelengths $\lambda_{2}$ and $\lambda_{3}$ and amplitudes $H_{2}$ and $H_{3}$, respectively. Upon invoking two equations involving lattice mismatch across compositional interfaces and one equation expressing global force balance in direction parallel to the interfaces, we obtain

$$
\begin{aligned}
\epsilon_{1}-\epsilon_{2}+H_{2}^{2} \frac{2 \pi^{2}}{\lambda_{2}^{2}} & =\epsilon_{m i s}^{(2)} \\
\epsilon_{1}-\epsilon_{3}+H_{3}^{2} \frac{2 \pi^{2}}{\lambda_{3}^{2}} & =\epsilon_{m i s}^{(3)} \\
\epsilon_{1}+\alpha_{2} \beta_{2} \epsilon_{2}+\alpha_{3} \beta_{3} \epsilon_{3} & =0 .
\end{aligned}
$$

The in-plane strain, bending, and substrate interaction energies are now given by

$$
\begin{aligned}
E_{\text {strain }} & =\frac{Y h W L}{2}\left(\epsilon_{1}^{2}+\alpha_{2} \beta_{2} \epsilon_{2}^{2}+\alpha_{3} \beta_{3} \epsilon_{3}^{2}\right) ; \\
E_{\text {bend }} & =\frac{Y h^{3} W L \pi^{4}}{3}\left(\frac{\alpha_{2} \beta_{2} H_{2}^{2}}{\left(1-\nu_{2}^{2}\right) \lambda_{2}^{4}}+\frac{\alpha_{3} \beta_{3} H_{3}^{2}}{\left(1-\nu_{3}^{2}\right) \lambda_{3}^{4}}\right) ; \\
E_{\text {sub }} & =\frac{k_{\text {eff }} L W}{2}\left(\alpha_{2} \beta_{2} H_{2}^{2}+\alpha_{3} \beta_{3} H_{3}^{2}\right) .
\end{aligned}
$$

Now, in the case of free-standing ternary superlattices, we first solve for the elastic strains $\epsilon_{1}$, $\epsilon_{2}$, and $\epsilon_{3}$ using Eqns. [19], subsequently minimize the total energy $E_{\text {total }}=E_{\text {strain }}+E_{\text {bend }}$ with respect to $H_{2}$ and $H_{3}$, and set $\lambda_{2} \approx \lambda_{3} \approx W$ to yield

$$
E_{\text {total }} \approx \frac{h^{3} L Y \pi^{2}}{W}\left[\frac{\alpha_{2} \beta_{2} \epsilon_{m i s}^{(2)}}{6\left(1-\nu_{3}^{2}\right)}+\frac{\alpha_{3} \beta_{3} \epsilon_{m i s}^{(3)}}{6\left(1-\nu_{2}^{2}\right)}\right] .
$$

We note that $E_{\text {total }} \sim 1 / W$ in the case of free-standing ternary superlattices, similar to freestanding binary superlattices. 
In passing, we also note that

$$
\epsilon_{2}=\frac{-\epsilon_{m i s}^{(2)}}{1+\alpha_{2} \beta_{2}}+\frac{\left[\alpha_{3} \beta_{3} h^{2}+12 H_{2}^{2}\left(1-\nu_{3}^{2}\right)\right] \pi^{2}}{6 W^{2}\left(1+\alpha_{2} \beta_{2}\right)\left(1-\nu_{3}^{2}\right)} .
$$

From Eq. [24], it is clear that $\alpha_{3} \beta_{3}$ cannot be too large $\left(\alpha_{3} \beta_{3} \lesssim W^{2} / h^{2}\right)$, otherwise $\epsilon_{2}$ becomes positive, which implies that stripe 2 is under tension and $H_{2}=0$ (contradicting our working assumption). Due to the similar Young's moduli of the TMDs, however, enormous $\alpha_{3} \beta_{3}$ values can only be realized when $\alpha_{3} \gg 1$, which physically means that the system can be approximately considered as a homogeneous one consisting of stripe 3 alone. Therefore, we will ignore these uninteresting cases and assume that $\alpha_{3} \beta_{3}$ is reasonable.

In the case of substrate-supported ternary superlattices, on the other hand, we minimize $E_{\text {total }}=$ $E_{\text {strain }}+E_{\text {bend }}+E_{\text {sub }}$ with respect to $H_{2}, H_{3}, \lambda_{2}$, and $\lambda_{3}$ to yield (while still assuming a non-zero wrinkling amplitude via $\left.k_{\text {eff }} \ll\left(\epsilon_{m i s}^{(2)}\right)^{2} Y / h\right)$

$$
\begin{aligned}
\lambda_{2}= & \pi \sqrt[4]{\frac{2 h^{3} Y}{3 k_{e f f}\left(1-\nu_{2}^{2}\right)}} ; \\
\lambda_{3}= & \pi \sqrt[4]{\frac{2 h^{3} Y}{3 k_{e f f}\left(1-\nu_{3}^{2}\right)}} ; \\
E_{\text {total }}= & L W\left\{\alpha_{2} \beta_{2} \epsilon_{m i s}^{(2)} \sqrt{\frac{k_{e f f} Y h^{3}}{6\left(1-\nu_{2}^{2}\right)}}+\alpha_{3} \beta_{3} \epsilon_{m i s}^{(3)} \sqrt{\frac{k_{e f f} Y h^{3}}{6\left(1-\nu_{3}^{2}\right)}}-\frac{k_{e f f} h^{2}}{12}\left[\frac{\alpha_{3} \beta_{3}\left(1+\alpha_{3} \beta_{3}\right)}{\left(1-\nu_{3}^{2}\right)}\right.\right. \\
& \left.\left.+\frac{2 \alpha_{2} \beta_{2} \alpha_{3} \beta_{3}}{\sqrt{\left(1-\nu_{2}^{2}\right)\left(1-\nu_{3}^{2}\right)}}+\frac{\alpha_{2} \beta_{2}\left(1+\alpha_{2} \beta_{2}\right)}{\left(1-\nu_{2}^{2}\right)}\right]\right\} .
\end{aligned}
$$

Again, $E_{\text {total }} \sim W$ in the case of substrate-supported ternary superlattices, similar to substratesupported binary superlattices.

Let us next consider the effect of misfit dislocations in a ternary superlattice, as illustrated in Fig. S9. Now, in the case of free-standing superlattices, the total energy becomes

$$
\begin{aligned}
E_{\text {total }} \approx & \frac{h^{3} L Y \pi^{2}}{W}\left[\frac{\alpha_{2} \beta_{2}\left(\epsilon_{m i s}^{(2)}-\rho_{12} b\right)}{6\left(1-\nu_{3}^{2}\right)}+\frac{\alpha_{3} \beta_{3}\left(\epsilon_{m i s}^{(3)}+\rho_{13} b\right)}{6\left(1-\nu_{2}^{2}\right)}\right] \\
& +\left(\left|\rho_{23}\right|+\left|\rho_{13}\right|+\left|\rho_{12}\right|\right) L E_{d i s},
\end{aligned}
$$

while in the case of substrate-supported ternary superlattices,

$$
\begin{aligned}
E_{\text {total }} \approx & L W\left\{\alpha_{2} \beta_{2}\left(\epsilon_{m i s}^{(2)}-\rho_{12} b\right) \sqrt{\frac{k_{\text {eff }} Y h^{3}}{6\left(1-\nu_{2}^{2}\right)}}+\alpha_{3} \beta_{3}\left(\epsilon_{m i s}^{(3)}+\rho_{13} b\right) \sqrt{\frac{k_{\text {eff }} Y h^{3}}{6\left(1-\nu_{3}^{2}\right)}}\right. \\
& \left.-\frac{k_{\text {eff }} h^{2}}{12}\left[\frac{\alpha_{3} \beta_{3}\left(1+\alpha_{3} \beta_{3}\right)}{\left(1-\nu_{3}^{2}\right)}+\frac{2 \alpha_{2} \beta_{2} \alpha_{3} \beta_{3}}{\sqrt{\left(1-\nu_{2}^{2}\right)\left(1-\nu_{3}^{2}\right)}}+\frac{\alpha_{2} \beta_{2}\left(1+\alpha_{2} \beta_{2}\right)}{\left(1-\nu_{2}^{2}\right)}\right]\right\} \\
& +\left(\left|\rho_{23}\right|+\left|\rho_{13}\right|+\left|\rho_{12}\right|\right) L E_{\text {dis }} .
\end{aligned}
$$

Given that the variations in $E_{\text {dis }}$ and Burger's vector $b$ are small across the different TMDs, we will take them to be constant here. Furthermore, $\rho_{23}+\rho_{12}+\rho_{13}=0$ due to the periodic boundaries. [Recall that, as discussed in the main text, $\rho_{i j}$ denotes the linear density of dislocations along 


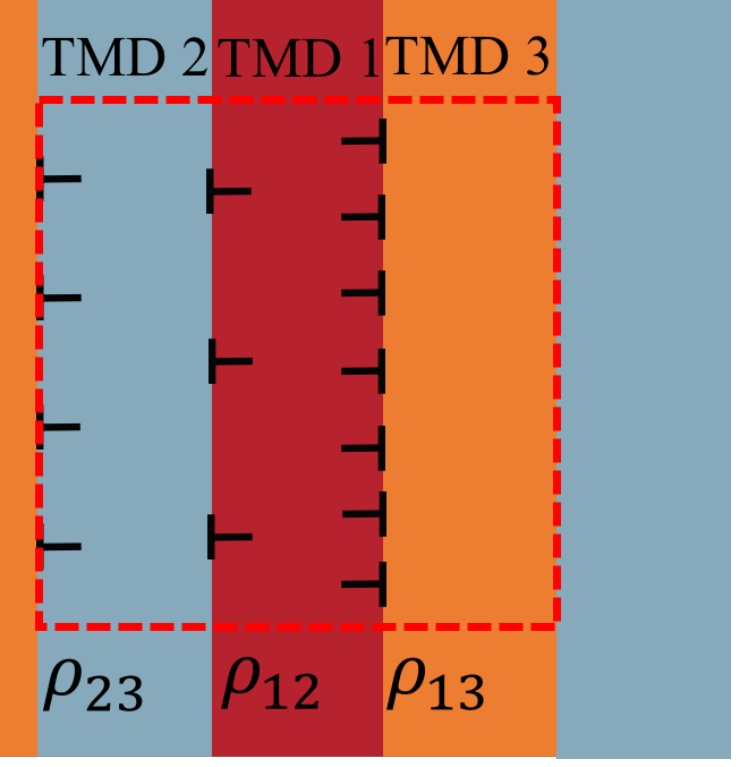

Figure S9: Schematic of unit cell and dislocation distribution in a ternary superlattice.

the interfaces between TMDs $i$ and $j$, with the convention that dislocation lines come out of the page such that $\rho_{i j}>0$ for dislocations with Burger's vector pointing downwards.] Finally, upon defining $C_{12}=h^{3} b Y \pi^{2} \frac{\alpha_{2} \beta_{2}}{6 W\left(1-\nu_{3}^{2}\right)}$ and $C_{13}=h^{3} b Y \pi^{2} \frac{\alpha_{3} \beta_{3}}{6 W\left(1-\nu_{2}^{2}\right)}$ for free-standing cases, while $C_{12}=b W \alpha_{2} \beta_{2} \sqrt{\frac{k_{e f f} Y h^{3}}{6\left(1-\nu_{2}^{2}\right)}}$ and $C_{13}=b W \alpha_{3} \beta_{3} \sqrt{\frac{k_{e f f} Y h^{3}}{6\left(1-\nu_{3}^{2}\right)}}$ for substrate-supported ones, Eqns. [28] and [29] above reduce to Eq. (10) in the main text.

\section{References}

${ }^{1}$ Shen, C.; Wang, Y. Acta Mater. 2003, 51, 2595-2610.

${ }^{2}$ Wang, Y. U.; Jin, Y. M.; Khachaturyan, A. G. J. Appl. Phys. 2002, 92 1351-1360.

${ }^{3}$ Khachaturyan, A. Theory of Structural Transformations in Solids, Dover Publications, 2013.

${ }^{4}$ Zou, X.; Liu, Y.; Yakobson, B. I. Nano Lett. 2013, 13, 253-258.

${ }^{5}$ Cakir, D.; Peeters, F. M.; Sevik, C. Appl. Phys. Lett. 2014, 104, 203110.

${ }^{6}$ Duerloo, K.-A. N.; Li, Y.; Reed, E. J. Nat. Comm. 2014, 5, 4214.

${ }^{7}$ Xie, S.; Tu, L.; Han, Y.; Huang, L.; Kang, K.; Lao, K. U.; Poddar, P.; Park, C.; Muller, D. A.; DiStasio, R. A.; Park, J. Science 2018, 359, 1131-1136. 